\title{
Elastic behaviour of the carotid artery in intact spontaneously hypertensive rats
}

\author{
D. Hayoz, B. Rutschmann, C. Y. Genton*, M. Niederberger, H. R. Brunner and B. Waeber
}

\begin{abstract}
Division of Hypertension, University Hospital, Lausanne, Switzerland, and *Institute of Pathology, University Hospital, Lausanne, Switzerland
\end{abstract}

\begin{abstract}
KEY WORDS: Arterial compliance, arterial distensibility, vascular hypertrophy, antihypertensive treatment.
Intact spontaneously hypertensive rats (SHR) were studied to assess the effect of prolonged antihypertensive treatment on the elastic behaviour of the external carotid artery. Thirty-week-old SHR received the ACE inhibitor captopril, the ateriolar dilator hydralazine or their vehicle for 6 weeks. These rats were compared to normotensive, vehicle treated WKY rats. The internal diameter of the carotid artery was measured continuously in halothane-anaesthetized rats using an echo-tracking device, and intra-arterial pressure was also monitored continuously, on the controlateral side. Captopril-and hydralazinetreated SHR as well as normotensive controls had similar blood pressure values. No significant shift in the distensibilitypressure curves was observed among vehicle-treated SHR and WKY rats or the SHR which had received captopril or hydralazine. Histological examination of the carotid artery fixed ex vivo with paraformaldehyde showed a significant increase in cross-sectional area in vehicle-treated SHR as compared to their normotensive counterparts. These results therefore suggest that the elastic behaviour of elastic arteries is not necessarily altered by the structural changes developing in response to hypertension.
\end{abstract}

\section{Introduction}

Large arteries undergo hypertrophy during chronic elevation in blood pressure ${ }^{[1]}$. These structural changes probably represent an important adaptive response allowing wall stress to remain constant ${ }^{[2]}$. Increased vascular thickness is expected to decrease the buffering function of conduit vessels. In fact, carotid arteries of spontaneously hypertensive rats (SHR) studied in vitro exhibit a reduced compliance ${ }^{|3|}$. Carotid artery pressure-compliance curves have also been established in situ in $\mathrm{SHR}^{|4|}$. Compared with normotensive controls, hypertensive animals showed a decreased arterial compliance only at intramural pressures lower than operating pressures. Recently, we developed an ultrasonic device making it possible to determine the compliance- or distensibility-pressure curves in vivo ${ }^{|5|}$. No significant shift in these curves could be seen in 16-week-old anesthaetized SHR, suggesting that the elastic behaviour of the carotid artery is not necessarily altered by chronic hypertension ${ }^{[6]}$.

The present investigations were undertaken to characterize the mechanical properties of the carotid artery of 36week-old SHR, either untreated or having received orally the angiotensin converting enzyme (ACE) inhibitor captopril or the arterial dilator hydralazine.

\section{Methods}

EQUIPMENT AND PROCEDURES

Ten 30-week-old normotensive male rats (WKY) and 27 age- and sex-matched spontaneously hypertensive rats (SHR) were obtained from Iffa Credo (L'Arbresle, France). The SHR were allocated to 6 weeks' treatment

Correspondence: Bernard Waeber. MD. Hypertension Division. CHUV. 1011 Lausanne. Switzerland. with either captopril $(n=7)$, hydralazine $(n=10)$ or vehicle (tap water, $n=10$ ). The drugs were administered in drinking water (captopril, $25 \mathrm{mg} / 30 \mathrm{ml}$ of water and hydralazine, $5 \mathrm{mg} / 30 \mathrm{ml}$ of water). The WKY rats were vehicle-treated. The animals were housed in a room with a constant temperature of $23^{\circ} \mathrm{C}$. Food, in the form of dried pellets of ordinary rat chow, and tap water were provided ad libitum.

On the day of the experiment, anaesthesia was induced with ether and then maintained with fluothane $(1 \cdot 5 \%)$. The right external carotid artery was cannulated with a catheter (PE 50, Portex, London, U.K.) filled with a heparinized $0.9 \% \mathrm{NaCl}$ solution. Intra-arterial pressure and heart rate were then monitored using a computerized data acquisition system ${ }^{[7]}$. The internal diameter of the left external carotid artery was measured at the same time using an AMode ultrasonic echo-tracking device ${ }^{|5|}$. Ten successive diameter-pressure recordings were determined for each animal in a $5 \mathrm{~min}$ period and then averaged for analysis. The apparatus can measure variations in the internal diameter of the carotid artery with a precision close to one micron. This degree of resolution is made possible by oversampling (5000 arterial diameter measurements per second) and averaging automatically 16 consecutive values. For the recordings, a $10-\mathrm{MHz}$ probe is positioned perpendicularly over the artery avoiding direct contact with the skin. An ultrasonic gel is employed for signal transduction. Once the measurements had been taken the carotid catheter was removed, the artery tied and the animals returned to individual cages to recover from the anaesthetic.

Twenty-four hours after the initial investigation, the rats were again subjected to a light ether anaesthesia. The right external iliac artery was cannulated with a polyethelene catheter (PE-50) while a PE-10 catheter was introduced 
into the right femoral vein. Thereafter, the rats were placed in a plastic tube to restrict their movement and were left to wake up. Intra-arterial pressure and heart rate were measured 90-120 min after the end of anaesthesia, when both parameters were stable. The animals were then killed with a lethal i.v. dose of penthotal $(20 \mathrm{mg})$. This was immediately followed by an intra-arterial infusion of phosphate buffer ( $\mathrm{pH} \mathrm{7.2)} \mathrm{until} \mathrm{clear} \mathrm{perfusate} \mathrm{issued} \mathrm{from} \mathrm{the}$ femoral vein. An intra-arterial infusion of a $4 \%$ paraformaldehyde solution was then set up at a constant pressure of $100 \mathrm{mmHg}$. The left external carotid artery prepared ex vivo by this method was excised and processed for histological examination. Paraffin-embedded tissue blocks were sectioned at a thickness of $4 \mu \mathrm{m}$ and stained by H.E. and van Gierson-Elastin coloration to differentiate the arterial wall layers. Morphometric calculations were performed under optical microscopy with a 200 -fold magnification and micrometric grid. Six fields per section were analysed in a blinded fashion.

\section{ANALYSIS}

The simultaneous and continuous acquisition of measurement of internal arterial diameter and blood pressure were processed on-line to compute a diameter (or cross section)-pressure relationship. The latter was subsequently converted into a cross-sectional distensibilitypressure curve, this curve fitting best with an arctangent function first described by Langewouters ${ }^{[8]}$. Cross-sectional distensibility represents the arterial compliance normalized for the cross-section. For the statistical evaluation of the diameter-, and distensibility-pressure curves, a multivariate analysis was used, based on Hotelling T2, taking diameter or distensibility values at three arbitrarily defined blood pressures in the range of measured values. The different curves were established within operating pressures, the upper and lower limits representing the mean systolic and diastolic values for the groups respectively. The crosssectional area (CSA) of the fixed arteries was determined according to the formula: $\mathrm{CSA}=[$ (internal radius + thickness $)^{2-}$ (internal radius) ${ }^{2}$. The between-groups comparison of body weight, mean blood pressure and heart rate measurements was made with a one-way analysis of variance, followed, when required, with Fisher's test for least significant differences. The data on cross-sectional area were evaluated using the Sheffe's test. Differences were considered significant for $P$ values $<0 \cdot 05$. Results are reported as means + SEM.

\section{Results}

On the day of the experiment, the body weight of vehicle-treated WKY rats was significantly greater than that of all groups of SHR (Table 1). Vehicle-treated SHR weighed more than corresponding animals given captopril $(P<0.05)$. Mean blood pressures measured in the conscious rats were significantly higher in control SHR than in WKY rats and in SHR having received captopril or hydralazine. The heart rate was similar in the four groups of animals.

Figure 1 illustrates the diameter-pressure relationship determined under halothane anaesthesia. For a given level of intra-arterial pressure, the internal diameter of the carotid artery tended to be larger in vehicle-treated SHR than in the three other groups of animals, but the difference was not significant.

The cross-sectional distensibility-pressure curves of the four study groups are displayed in Fig. 2. The curve obtained in captopril- and hydralazine-treated SHR appeared to be the direct continuation of that established in control SHR. Cross-sectional distensibility was slightly higher in SHR (whether treated or untreated) than in normotensive WKY rats, but the difference did not achieve a significant level.

The cross-sectional area of the carotid artery of vehicletreated SHR was significantly greater than that of normotensive WKY controls (Fig. 3). Administration of captopril or hydralazine to SHR reduced the cross-sectional area, rendering the difference in this parameter between treated SHR and WKY rats not significant.

\section{Discussion}

The mechanical properties of the arterial wall can be characterized by measuring arterial compliance, which is defined as the blood volume stored or released given a specific change in intraluminal pressure, as well as the cross-section adjusted distensibility ${ }^{|9|}$. These parameters were measured here in intact rats by relating variations in the internal diameter to intra-arterial pressure monitored simultaneously at the carotid artery. The distensibility-pressure curve is non-linear, the distensibility decreasing as blood pressure increases. Determination of the arterial diameter was achieved by means of a noninvasive, high-precision echo-tracking device.

Hypertension is known to induce thickening in the arterial wall and to accelerate the development of atheroscle-

Table 1 Characteristics of the study groups (means \pm SEM)

\begin{tabular}{llll}
\hline & BW(g) & MBP $(\mathrm{mmHg})$ & HR (beat.min $\left.{ }^{-1}\right)$ \\
\cline { 1 - 2 } Vehicle-treated WKY rats & $403 \pm 5^{*}$ & $145 \pm 2$ & $422 \pm 9$ \\
Vehicle-treated SHR & $375 \pm 7 \#$ & $185 \pm 4^{* *}$ & $425 \pm 11$ \\
Captopril-treated SHR & $339 \pm 10$ & $154 \pm 6$ & $410 \pm 10$ \\
Hydralazine-treated SHR & $356 \pm 10$ & $138 \pm 9$ & $436 \pm 9$ \\
\hline
\end{tabular}

$\mathrm{BW}=$ body weight $; \mathrm{MBP}=$ mean blood pressure; $\mathrm{HR}=$ heart rate;

$* P<0.05 ; * * P<0.01$ vs other groups;

$\# P<0.05$ vs captopril. 


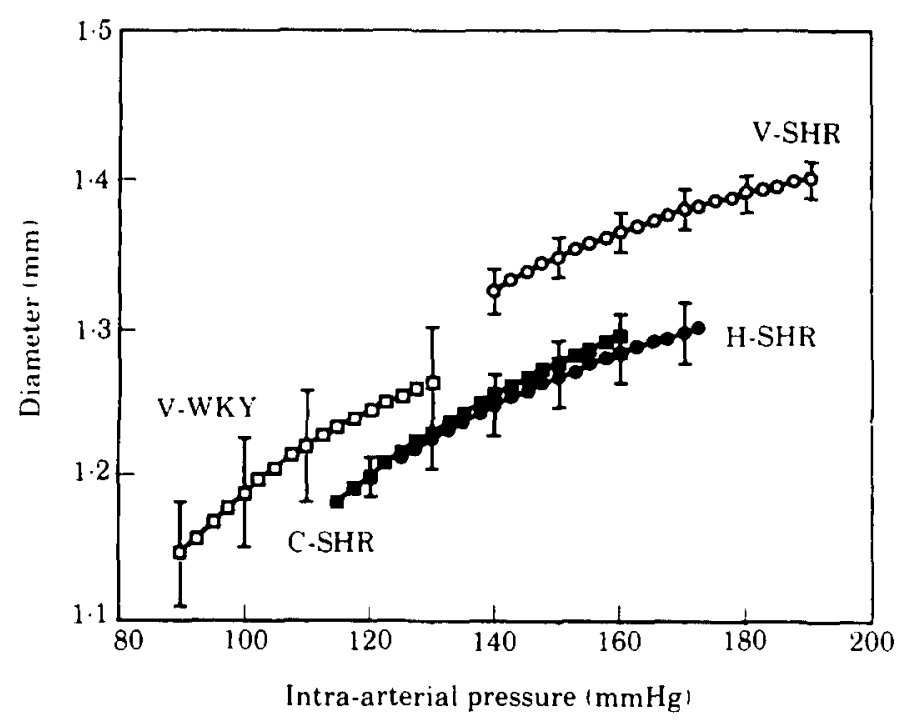

Figure I Line plot showing the relationship between intra-arterial pressure and the internal diameter (mean \pm SEM) of the carotid artery in vehicle-treated WKY rats (V-WKY), vehicle-treated SHR (V-SHR) and SHR having received captopril (C-SHR) or hydralazine (H-SHR).

rosis ${ }^{(1)}$. As anticipated, histological examination of the carotid artery revealed a larger cross-sectional area in genetically hypertensive rats (SHR) compared with normotensive controls (WKY rats). This difference in arterial wall structure could be expected to diminish the distensibility of the blood vessel. In fact, our findings showed, if anything, a trend towards an increase in arterial distensibility rather than a decrease, in hypertensive animals. Very similar observations were made recently in humans using the same echo-tracking device ${ }^{|6|}$. The distensibility of the radial artery, a muscular artery, appeared slightly better in untreated hypertensive patients than in age-matched normotensive subjects.

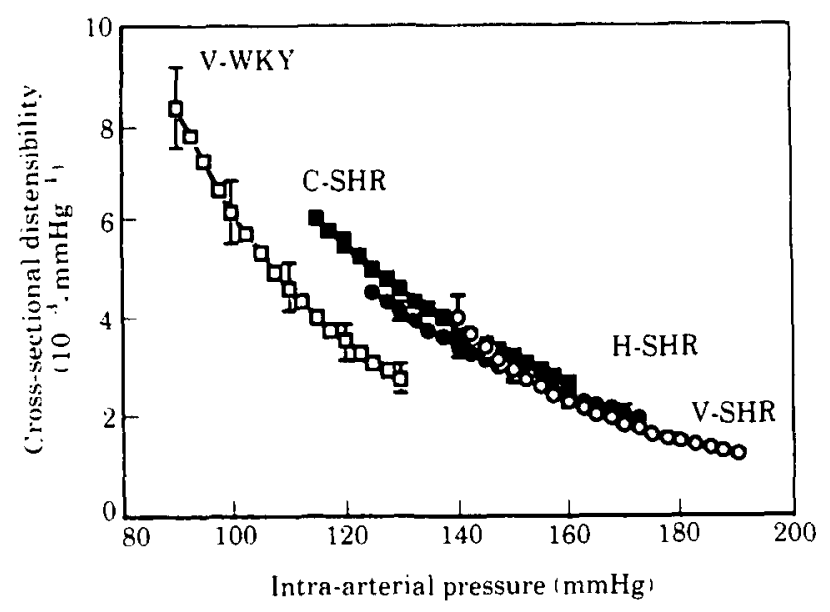

Figure 2 Line plot showing the relationship between intra-arterial pressure and cross-sectional distensibility (mean \pm SEM) of the carotid artery in vehicle-treated WKY rats (V-WKY), vehicletreated SHR (V-SHR) and SHR having received captopril (CSHR) or hydralazine (H-SHR).
One could argue that WKY rats are not an ideal control strain for SHR. It is therefore noteworthy that hypertensive animals treated with captopril or hydralazine behave, in terms of arterial distensibility, like untreated SHR. The lack of modification in arterial distensibility was seen despite a treatment-induced reduction in wall thickness. There was indeed no significant difference in crosssectional area between SHR having their blood pressure lowered by the ACE inhibitor or the vasodilator and normotensive WKY rats.

That morphological changes are not necessarily translated into modifications of the mechanical properties of the arterial wall may be regarded, at first glance, as very surprising. The reaction of the arterial wall to high blood pressure represents an adaptive process that tends to maintain an optimal wall tensile stress. The arterial wall exposed to

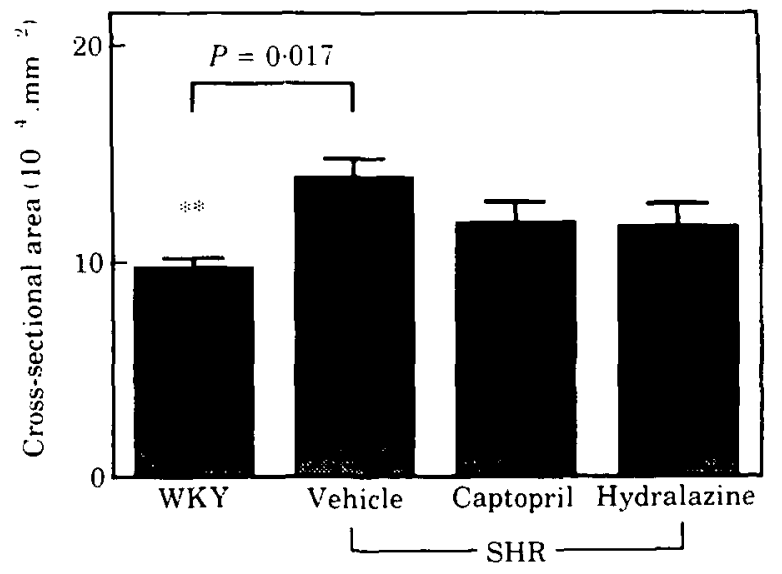

Figure 3 Cross-sectional area of carotid arteries (mean \pm SEM) fixed ex vivo in normotensive vehicle-treated WKY rats and SHR given either captopril, hydralazine or their vehicle. 
increased mechanical forces is subjected to a reorganization involving smooth muscle cells, elastic and collagen fibres ${ }^{[2]}$. The ratio, distribution and orientation of these elements influence substantially the elastic properties of the arterial wall. The structural changes associated with hypertension may therefore be associated with a decreased incremental elastic modulus, defined as the increase in wall stress for a given fractional increase in arterial diameter. Such an adaptive response could account for the fact that arterial distensibility is not reduced in hypertensive humans and animals.

In conclusion, the present data, obtained in intact rats show that hypertension-induced remodelling does not necessarily reduce arterial distensibility.

This work was supported by grants from the Swiss National Science Foundation, The Cardiovascular Research Fund and the Swiss Association of Cigarette Manufacturers.

\section{References}

[1] Folkow B. Cardiovascular structural adaption: its role in the initiation and maintenance of primary hypertension. Clin Sci Mol Med 1992; 55 (Suppl 4): 3-22.
[2] Glagov S, Vito R, Giddens DP, Zanins CK. Micro-architecture and composition of artery walls: relationship to location, diameter and the distribution of mechanical stress. J Hypertens 1992; 10 (Suppl 6): 101-4.

[3] Caputo L, Tedgui A, Poitevin P, Lévy BI. In vitro assessment of diameter-pressure relationship in carotid arteries from normotensive and spontaneously hypertensive rats. J Hypertens 1992; 10 (Suppl 6): 27-30.

[4] Benetos A, Bonaziz H, Albaladejo P, Lévy BU, Safar ME. Physiological and pharmacological changes in the carotid artery pressure-volume curve in situ in rats. J Hypertens 1992; 10 (Suppl 6): 127-31.

[5] Tardy Y, Meister JJ, Perret F, Brunner HR, Arditi M. Noninvasive estimate of the mechanical properties of peripheral arteries from ultrasonic and photoplethysmographic measurements. Clin Phys Physiol Meas 1991; 12: 39-54.

[6] Hayoz D, Rutschmann B, Perret F et al. Conduit artery compliance and distensibility are not necessarily reduced in hypertension. Hypertension 1992; 20: 1-6.

[7] Flückiger JP, Gremaud G, Waeber B et al. Measurement of sympathetic nerve activity in the unanesthetized rat. J Appl Physiol 1989; 67: 250-5.

[8] Langewouters GJ, Wesseling KH, Godehard WJA. The static elastic properties of 45 human thoracic and 20 abdominal aortas in vitro and the parameters of a new model. J Biomechanics 1984; 17: 425-35.

[9] O'Rourke MF, Brunner HR. Introduction to arterial compliance and function. J Hypertension 1992; 10 (Suppl 6): 3-5. 